\title{
Agreement of Single- and Multi-Frequency Bioimpedance Measurements in Hemodialysis Patients: An Ancillary Study of the Frequent Hemodialysis Network Daily Trial
}

\author{
Jochen G. Raimann ${ }^{a}$ Samer R. Abbas ${ }^{a} \quad$ Li Liu $^{\mathrm{a}, \mathrm{e}} \quad$ Fansan Zhu ${ }^{\mathrm{a}}$ Brett Larive ${ }^{\mathrm{b}}$ \\ Peter Kotanko $^{a}$ Nathan W. Levin ${ }^{a}$ George A. Kaysenc, d for the FHN Trial \\ ${ }^{a}$ Research Division, Renal Research Institute, New York, N.Y., ${ }^{b}$ Department of Quantitative Health Sciences, \\ Cleveland Clinic, Cleveland, Ohio, and 'Division of Nephrology, Department of Medicine, and d Department of \\ Biochemistry and Molecular Medicine, University of California Davis, Davis, Calif., USA; ${ }^{e}$ Renal Division, \\ Peking University First Hospital, Beijing, PR China
}

\section{Key Words \\ Bioimpedance measurements - Hemodialysis . \\ Frequent Hemodialysis Network Daily Trial · \\ Multi-frequency bioimpedance spectroscopy . \\ Single-frequency bioimpedance analysis}

\begin{abstract}
Background: Bioimpedance analysis (BIA) is well established to assess body composition. Agreements between singleand multi-frequency bioimpedance (SF-BIA, MF-BIS) measurements in subjects undergoing 6 or 3 times/week hemodialysis (HD) were analyzed. Methods: Total body water (TBW) and intra- and extracellular fluid (ICF, ECF) of subjects enrolled in the Frequent Hemodialysis Network (FHN) Daily Trial (www.clinicaltrials.gov No. NCT00264758) were measured with a Hydra 4200 at baseline (BL) and at 5 months (M5). Volumes were computed using SF (at $50 \mathrm{kHz}$ ) and MF approaches. Agreement was assessed by means of linear regression and Bland-Altman analysis and treatment effects by t test. Results: 35 subjects ( 17 on the more frequent regimen, 26 males, 20 African-American, $48 \pm 9$ years, pre-HD weight $84 \pm 19 \mathrm{~kg}$ ) were studied. Assessments with SF-BIA and MFBIS correlated significantly at BL and M5 in both arms. No
\end{abstract}

proportional errors, but systematic biases over the entire range of values were found at BL and M5. Agreement did not differ between subjects randomized to either HD treatment arm at both time points. MF-BIS appears to have better precision than SF-BIA allowing the observation of a significant treatment effect by the intervention $[-1.5(95 \% \mathrm{Cl}-2.5$ to -0.5) I] on ECF, not found for ECF SF-BIA. Precision also affected the statistical power of the SF-BIA data in the current analysis. Conclusion: Both methods showed agreement without significant proportional errors regardless of HD frequency and can be used for longitudinal analyses. SF-BIA has lower precision which needs thorough consideration in the design of future trials with similar outcomes.

(c) 2014 S. Karger AG, Basel

\section{Introduction}

Bioimpedance analysis (BIA) is a portable and relatively inexpensive tool for the assessment of body composition. A high level of reliability and good agreement to 'gold standard methods' has been reported for single-frequency BIA (SF-BIA) and multi-frequency bioimpedance spectroscopy (MF-BIS) [1-5].

\section{KARGER}

E-Mail karger@karger.com

www.karger.com/nec
(C) 2014 S. Karger AG, Basel

$1660-2110 / 14 / 1282-0115 \$ 39.50 / 0$
Jochen G. Raimann

315 East 62nd Street

Suite 4-05

New York, NY 10065 (USA)

E-Mail Jochen.Raimann@ rriny.com 
Regression models, developed based on data from validation studies using 'gold standard' methods such as magnetic resonance imaging, DEXA or isotope dilution, are used for the estimation of body composition of the body segment of interest using the collected bioimpedance data. The aforementioned good agreement to 'gold standard methods' is true for the method the regression model has been developed with, but may very well differ from other approaches based on other methods. Recent evidence suggests that at the current state-of-the-art after no absolute correct measure [particularly for the assessment of extracellular fluid volume (ECF)] may exist, since different dilution markers provide different volumes of distribution $[5,6]$.

Most commonly used is the whole-body bioimpedance technique, which measures whole-body resistance and reactance and thus allows the estimation of total body water (TBW), ECF and intracellular fluid (ICF). From the technical point of view bioimpedance can be separated into two main categories: methods using one frequency only (most commonly 5, 50 or $100 \mathrm{kHz}$ ) and those using multiple frequencies (mostly in the range from 5 to $1,000 \mathrm{kHz}$ ).

The frequency determines whether the current penetrates the cells or remains in the extracellular compartment. Low frequencies pass through the extracellular compartment, whereas higher frequencies penetrate the cells. This allows the assessment of the resistance of both extra- and intracellular compartments [3]. By using the well-established Cole model, it is possible to distinguish extra- and intracellular resistance in the spectrum of frequencies [7]. Kotler and Wang have published models designed for SF-BIA and De Lorenzo for MF-BIS; however, both studied patient populations did not include dialysis patients [3]. Evidence shows MF-BIS to be more accurate when compared to SF-BIA in determining fluid volumes $[8,9]$.

Differences in body composition have also been reported to affect the accuracy and precision of bioimpedance methods for fluid volume estimation [10]. Accordingly, interventions which alter fluid overload and body hydration may potentially affect the accuracy and precision of used measurement techniques by introducing bias in the measurement. This may also affect the ability of a method to detect changes of either fluid volume over a defined period of time, as was for example reported for the comparison between whole-body and segmental MF-BIS [11].

More frequent hemodialysis (HD) has been reported to affect interdialytic weight gains [12] and extracellular volume assessed by single-frequency bioimpedance [13]. To the best of the authors' knowledge, the potential bias introduced by a change in frequency of HD has not yet been investigated in detail.

The primary aim of this study was to investigate whether more frequent HD affects the agreement between the assessment of intra- and extracellular volume and TBW during two different regimens of dialysis frequency using two technically and conceptually distinguished bioimpedance approaches: SF-BIA and MF-BIS. As the secondary aim, both bioimpedance methods were used for the assessment of the treatment effect of increased HD frequency on all measured fluid volumes, ultrafiltration volume and pre-HD weights. The tertiary aim was the determination of the statistical power of the treatment effect analysis with either method.

\section{Methods}

All patients enrolled in the FHN Daily Trial (www.clinicaltrials. gov No. NCT00264758) from 07/2006 to 03/2010 at nine of the participating centers were approached for participation in this ancillary study to the main trial. Patients were enrolled at the University of California, Davis, part of the California Consortium, and dialysis clinics of the Renal Research Institute (RRI) in the urban area of New York City, part of the RRI consortium (see appendix and Sergeyeva et al. [14]). A detailed overview of the inclusion and exclusion criteria of the main trial has been published [15]. The study was approved by the institutional review board of Beth Israel Medical Center and University of California Davis, and was conducted according to the Declaration of Helsinki. All patients signed informed consent.

\section{Study Design}

This ancillary study followed enrolled subjects during the entire active study period in the prospective, randomized, controlled FHN Daily Trial. Patients enrolled in the main trial were randomized on a facility level to receive either more frequent (6 times/week) or a conventional (3 times/week) HD regimen. Due to the randomization on a facility base and the approach of all patients enrolled in the main trial, the study population of this ancillary study can be considered as being randomized to treatment arm allocation.

\section{Measurements}

Bioimpedance spectroscopy measurements were performed before dialysis treatment prior to randomization at baseline and after 5 months into the trial by trained personnel. Measurements were conducted after patients were in a supine position for a minimum of $10 \mathrm{~min}$. Electrodes attached at wrist and ankle measured intraand extracellular resistance serving the computation of extra- and intracellular volume. All measurements were performed using the Hydra 4200 Bioimpedance Analyzer (San Diego, Calif., USA) at the same time and the data were saved electronically for analyses.

\section{Bioimpedance}

Multi-Frequency Bioimpedance Spectroscopy

Measurements of resistance, reactance and impedance at frequencies in the range from 5 to $1,000 \mathrm{kHz}$ were used for the calculation of extra- and intracellular resistance using the Cole model 

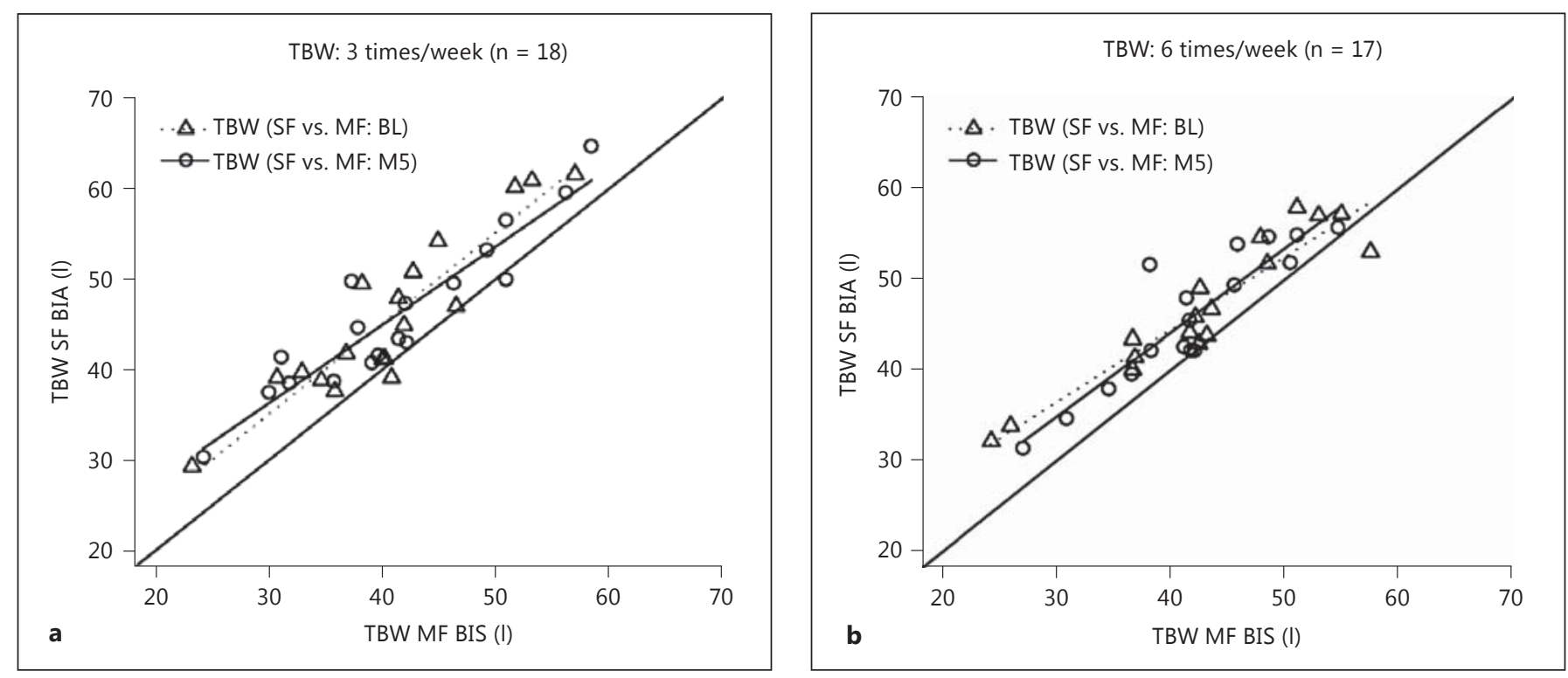

Fig. 1. Regression analysis of TBW as estimated per single- and multi-frequency (SF; MF) bioimpedance in patients randomized in $\mathbf{a}$ the conventional ( 3 times/week HD; $\mathrm{n}=18$ ) and $\mathbf{b}$ the interventional (more frequent HD 6 times/week HD; $\mathrm{n}=17$ ) arm at baseline and 5 months after randomization.

Table 1. Demographics of all study subjects

\begin{tabular}{lllll}
\hline & All patients & $\begin{array}{l}\text { Conventional } \\
\text { subjects }\end{array}$ & Daily subjects & p value \\
\hline Number & 35 & 18 & 17 & n/a \\
Age, years & $47.8 \pm 8.7$ & $48.5 \pm 10.8$ & $47.1 \pm 6.1$ & n.s. \\
Male/female & $26 / 9$ & $12 / 6$ & $14 / 3$ & n.s. \\
Black/non-Black & $15 / 20$ & $8 / 10$ & $7 / 10$ & n.s. \\
Pre-dialysis body weight, kg & $84.2 \pm 18.8$ & $85.8 \pm 18.7$ & $82.6 \pm 19.3$ & n.s. \\
Body mass index & $29.4 \pm 6.7$ & $30.9 \pm 7.0$ & $27.9 \pm 6.3$ & n.s. \\
\hline
\end{tabular}

The demographics of all enrolled study subjects who completed all measurements, a subset of subjects who were randomized to the more frequent arm (6 times/week HD), and those who were randomized to the conventional group ( 3 times/week HD) are shown. Continuous variables are displayed as mean $\pm \mathrm{SD}$, categorical variables as count in the respective category.
[7] with software provided by the manufacturer. Extra- and intracellular resistances were used for the computation of ECF and ICF using the model developed by De Lorenzo et al. [3] (see Appendix). TBW was calculated as the sum of ECF and ICF.

Single-Frequency Bioimpedance Analysis

Resistance and reactance data at $50 \mathrm{kHz}$ was extracted for the computation of TBW and intracellular water employing the models developed by Kotler et al. [16] and Wang et al. [17] (see Appendix). ECF was calculated as the difference between TBW and ICF.

\section{Statistical Analyses}

Data is reported as mean and standard deviation or $95 \%$ confidence intervals (CI), as appropriate. p value $<0.05$ were considered significant. All analyses were conducted with $\mathrm{R}$ version 3.0.3 ('Warm Puppy'; R Foundation for Statistical Computing; Vienna, Austria) [18] and the packages pwr and hydroGOF were used to perform the power calculation and the computation of the root mean square error (RMSE), respectively.

\section{Agreement between Methods}

As the primary aim, the assessment of agreement between methods for this analysis follows the approach by Altman and Bland [18] and the assessment of the methods' precisions: (1) regression plot and visual inspection of data; (2) assessment of relative bias (difference between the methods) and the associated error (standard deviation of this difference), and (3) the assessment of measurement errors proportional to the mean [19]. 


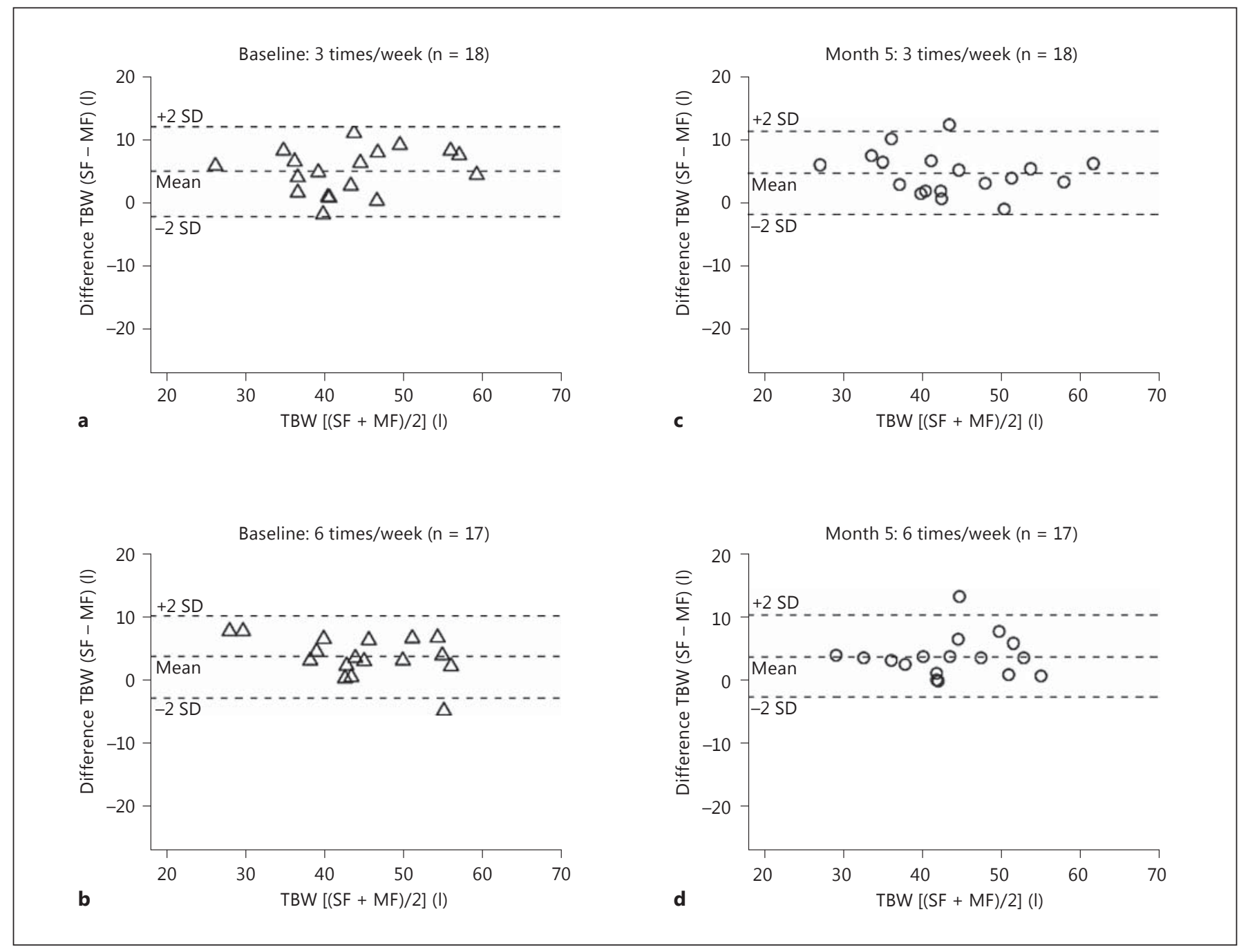

Fig. 2. Bland-Altman plot of TBW as estimated per single- and multi-frequency (SF; MF) bioimpedance in patients randomized in the conventional ( 3 times/week HD; $n=18$ ) and the interventional (more frequent HD 6 times/week HD; $\mathrm{n}=17)$ arm at baseline $(\mathbf{a}, \mathbf{b})$ and 5 months $(\mathbf{c}, \mathbf{d})$ after randomization.

\section{Treatment Effect}

The secondary aim, the treatment effect on body fluid volumes, ultrafiltration volume and pre-HD weight, between the study arms, was assessed following a two-step approach: First, the mean difference for each treatment arm between the volume estimation at month 5 and at baseline was calculated, and then, in a second step, the difference between the differences in the treatment arm was used to compare the effectiveness of the intervention of increasing the dialysis frequency. Comparisons are reported as means and $95 \%$ CI and were formally tested using paired and Welch's unpaired t test.

\section{Power Calculation}

Based on the precision obtained from the data presented in this work, we estimated the statistical power at which we are able to draw conclusions with regard to our results. The effect size was estimated based on the treatment effect and the error of each respective method. Based on this effect, size power and sample size, which would have been required in order to reach a power of $80 \%$, were estimated.

\section{Results}

Data on recruitment efficiency [14], baseline characteristics $[20,21]$ and on primary [12] and secondary outcomes $[13,22]$ of the FHN Daily Trial have been published previously. 48 patients were enrolled in the study. Of these, 9 subjects were withdrawn from the main trial and measurements of 4 subjects could not be analyzed 

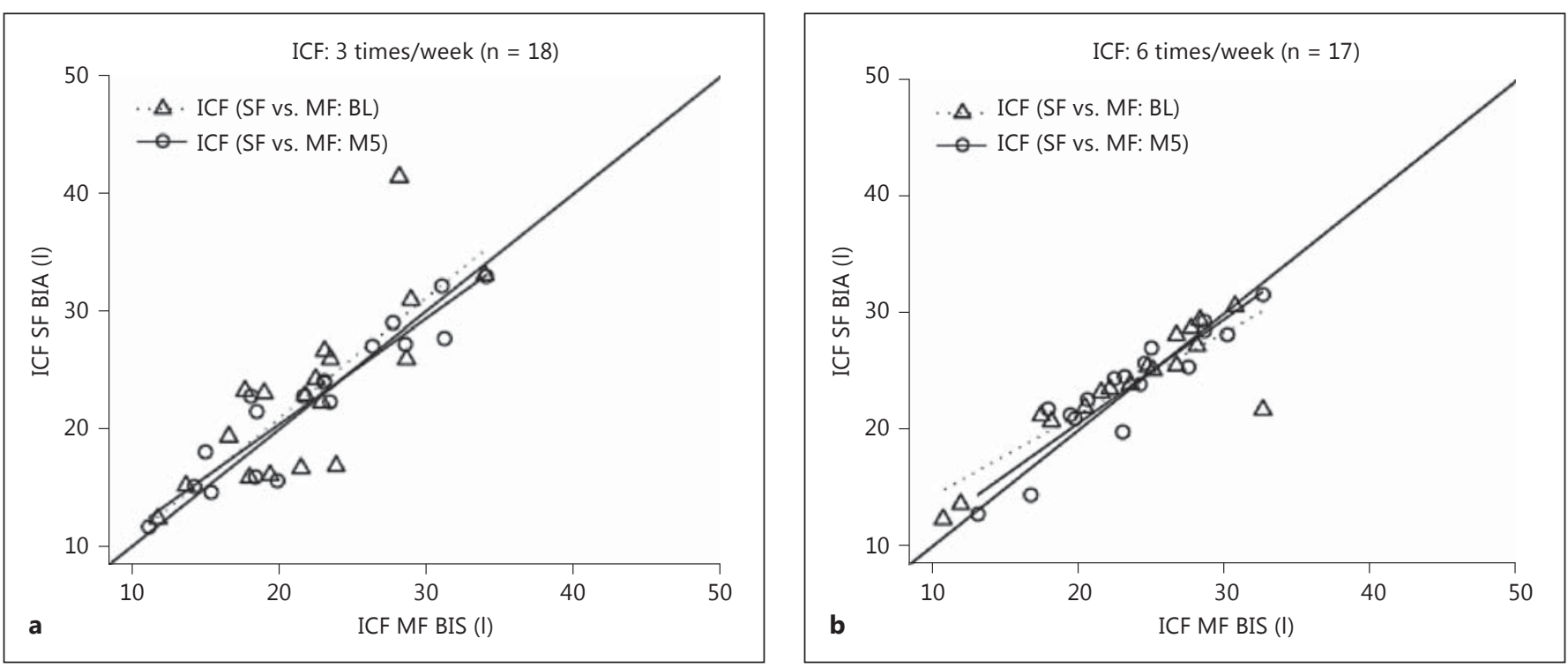

Fig. 3. Regression analysis of ICF as estimated per single- and multi-frequency (SF; MF) bioimpedance in patients randomized in $\mathbf{a}$ the conventional (3 times/week $\mathrm{HD} ; \mathrm{n}=18$ ) and $\mathbf{b}$ the interventional (more frequent HD 6 times/week; $\mathrm{n}=17$ ) arm at baseline and 5 months after randomization.

due to technical problems with the measurement data. Thus, 35 subjects (see table 1 for Demographics at Baseline) completed measurements at baseline and month 5 of the study and were included in this comparative analysis.

\section{Agreement between Methods}

Total Body Water

Measurement of TBW by both MF-BIS and SF-BIA values were correlated [in those randomized to the more frequent arm ( 6 times a week dialysis): $\mathrm{R}^{2}$ of 0.88 ( $\mathrm{p}<$ 0.01 ), and in those randomized to the conventional arm (3 times a week dialysis): $\mathrm{R}^{2}$ of $0.83(\mathrm{p}<0.01)$ ]. Figure 1 shows the corresponding regression plots. The data showed on average a significant systematic bias with SF yielding higher values than MF-BIS (table 3; fig. 2), but no proportional error was evident (fig. 2).

Among subjects who had been randomized to more frequent HD for 5 months, both MF-BIS and SF-BIA assessment of TBW correlated [more frequent: $\mathrm{R}^{2}$ of 0.80 $(\mathrm{p}<0.01)$; conventional: $\mathrm{R}^{2}$ of $\left.0.86(\mathrm{p}<0.01)\right]$ (fig. 1$)$. As observed at baseline, SF values were significantly greater (table 3; fig. 2), but without proportional error (fig. 2). The analysis of the agreement of TBW between both arms did not significantly differ from baseline or between the treatment arms following randomization (table 3) as shown by the treatment comparison (table 3 ).

FHN Ancillary Study: Comparative Studies of Whole-Body BIA and BIS
Intracellular Fluid

In regression analysis, ICF assessments using MF-BIS and SF-BIA correlated significantly [more frequent: $\mathrm{R}^{2}$ of $0.72(\mathrm{p}<0.01)$; conventional: $\mathrm{R}^{2}$ of $0.60(\mathrm{p}<0.01)$ ] (fig. 3 ). The values obtained with the SF method and MF method did not show a significant bias (table 3 ). No proportional error was observed in the data (fig. 4).

ICF MF-BIS and SF-BIA assessments after 5 months in those patients randomized to receive HD 6 times/week showed a significant correlation [more frequent: $\mathrm{R}^{2}$ of 0.85 $(\mathrm{p}<0.01)$; conventional: $\mathrm{R}^{2}$ of $\left.0.87(\mathrm{p}<0.01)\right]$ (fig. 3 ). Consistent with the baseline assessment, the SF values did not show a significant bias and did not show a proportional error (table 3; fig. 4). The treatment comparison showed that treatment allocation did not significantly affect the systematic bias between SF-BIA and MF-BIS (table 3).

\section{Extracellular Fluid}

ECF assessment with MF-BIS and SF-BIA correlated significantly [more frequent: $\mathrm{R}^{2}$ of $0.74(\mathrm{p}<0.01)$; conventional: $\mathrm{R}^{2}$ of $0.37(\mathrm{p}<0.01)$ ] (fig. 5). The values obtained with the SF method were systematically greater than those assessed with MF (table 3; fig. 6). No proportional error was observed in the data (fig. 6).

Five months into the study, SF-BIA values also correlated significantly [more frequent: $\mathrm{R}^{2} 0.61(\mathrm{p}<0.01)$; conventional: $R^{2} 0.61(p<0.01)$ ] (fig. 5). Values estimated 

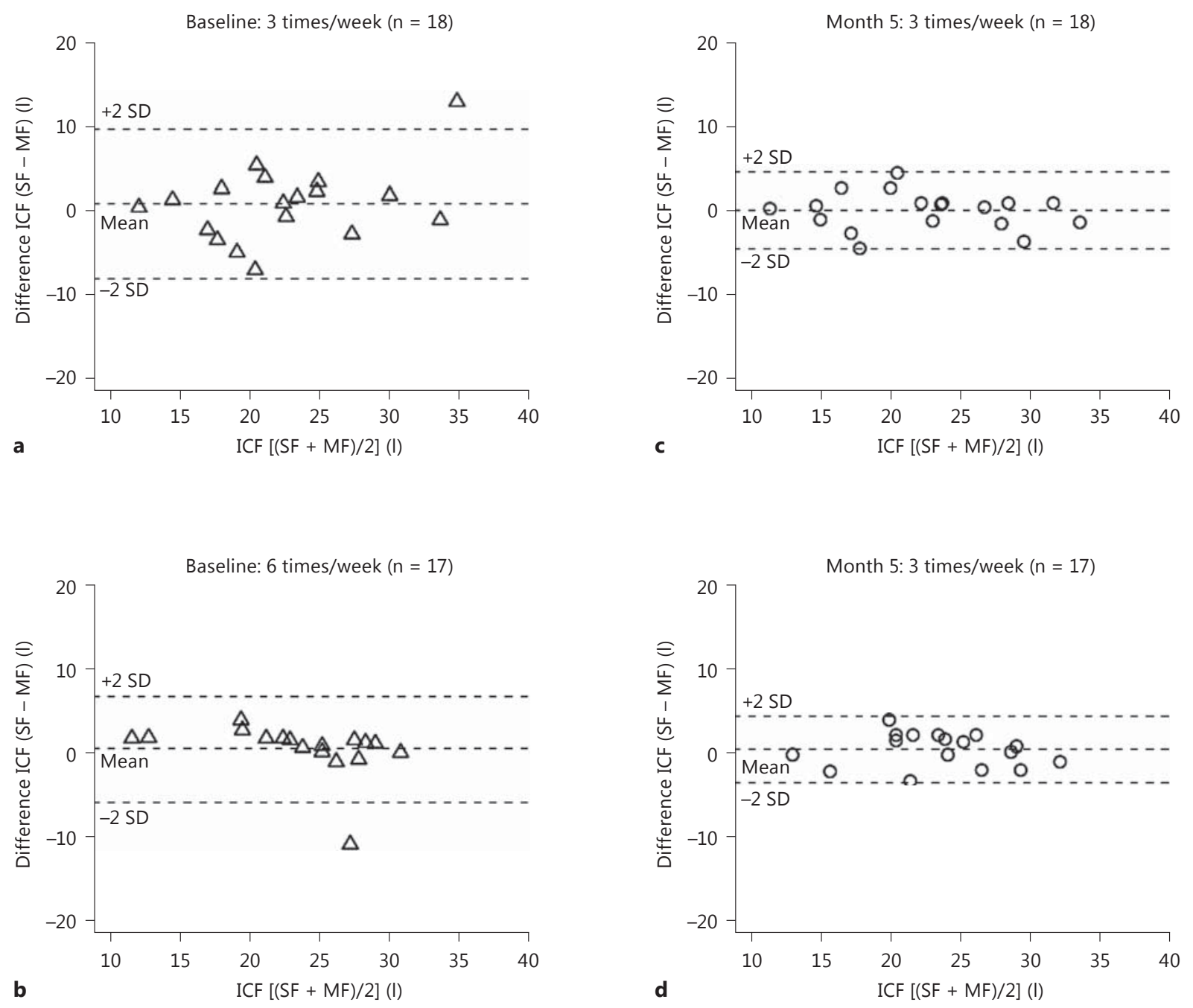

Fig. 4. Bland-Altman plot of ICF as estimated per single- and multi-frequency (SF; MF) bioimpedance in patients randomized in the conventional ( 3 times/week HD; $\mathrm{n}=18$ ) and the interventional (more frequent HD 6 times/ week HD; $\mathrm{n}=17)$ arm at baseline $(\mathbf{a}, \mathbf{b})$ and 5 months $(\mathbf{c}, \mathbf{d})$ after randomization.

with SF-BIA were again higher than MF-BIS values regardless of treatment allocation (table 3; fig. 6). The data showed no proportional error in Bland-Altman analysis (fig. 3). Treatment comparison showed that treatment allocation did not significantly affect the systematic bias between SF-BIA and MF-BIS (table 3). At both baseline and at month 5, the bias observed in TBW was similar to that observed in measurement of ECF.

\section{Treatment Effect of More Frequent Hemodialysis}

The comparison of the precision at each time point showed no treatment effects on the precision of all vol- ume estimations (reflected by the CIs at each time point in tables 2 and 3) using either measurement method (table 2 for univariable treatment effects of more frequent dialysis).

Measured by MF-BIS and SF-BIA, TBW showed a non-significant trend to decrease from baseline to month 5 of the study. It is of note that the treatment effect estimated by MF-BIS is larger in magnitude compared to SFBIA (table 2). ICF measured at month 5 by both methods was not significantly different from baseline (table 2) and no significant treatment effect by the intervention was found. 

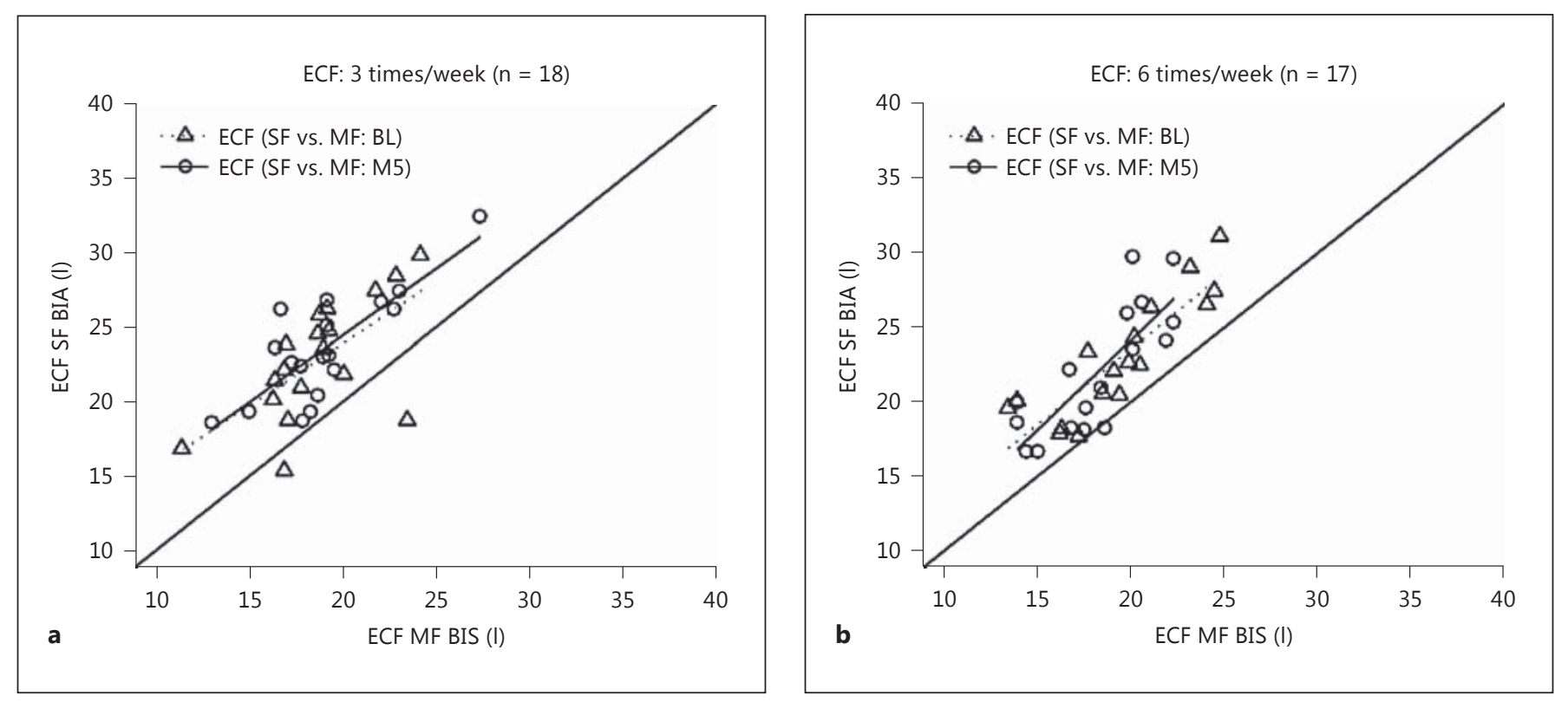

Fig. 5. Regression analysis of ECF as estimated per single- and multi-frequency (SF; MF) bioimpedance in patients randomized in $\mathbf{a}$ the conventional ( 3 times/week HD; $\mathrm{n}=18$ ) and $\mathbf{b}$ the interventional (more frequent HD 6 times/week HD; $\mathrm{n}=17$ ) arm at baseline and 5 months after randomization.

Table 2. Treatment effect of the more frequent HD (6 times/week as compared to 3 times/week) on TBW, extra- and intracellular volume as measured by single- and multi-frequency bioimpedance (SF, MF), ultrafiltration volume and pre-HD body weight of enrolled study subjects, who completed all measurements until month 5 (18 conventional subjects, 17 daily subjects)

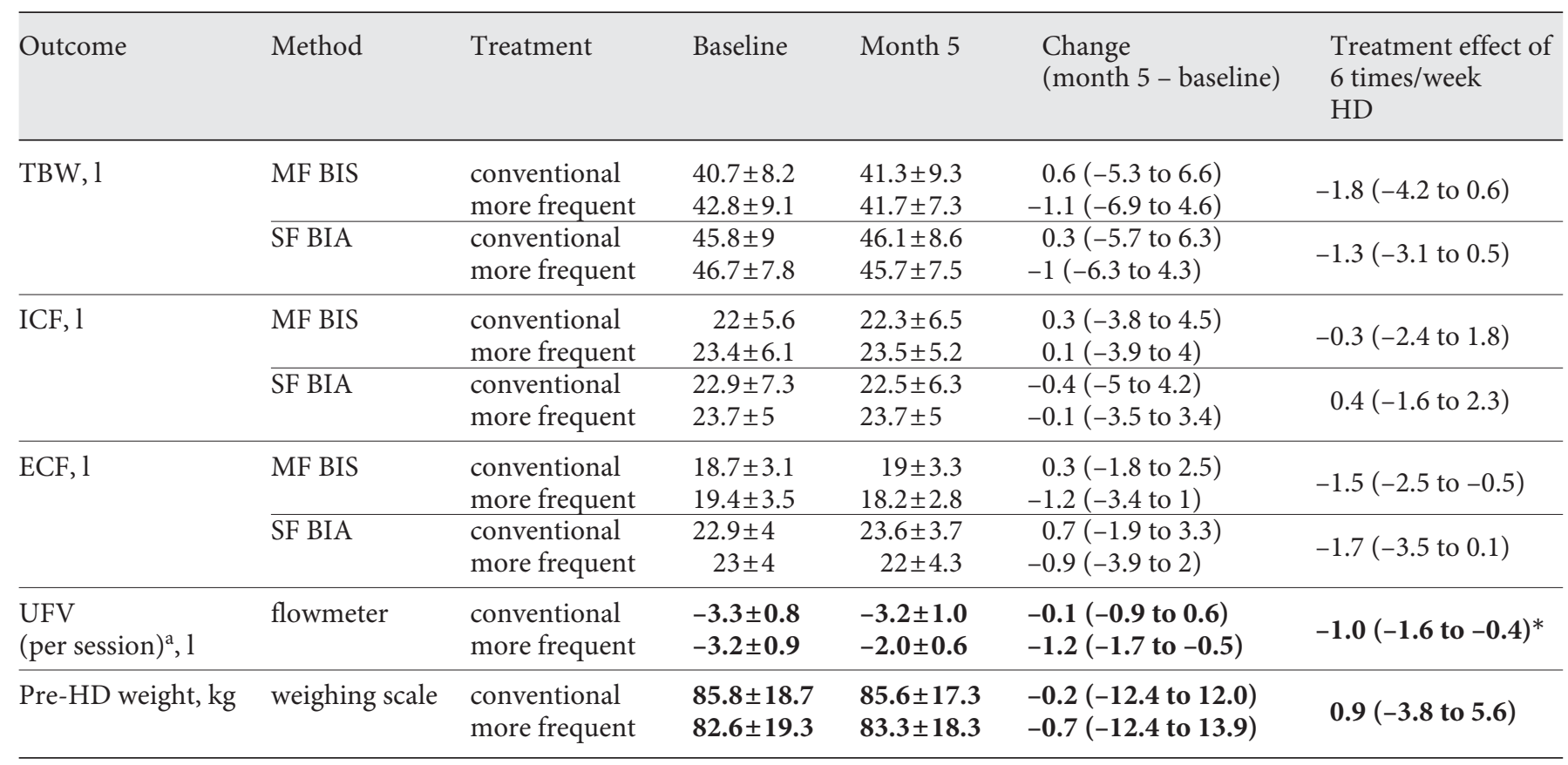

Data are presented as mean \pm SD or mean and $95 \%$ CI as indicated. * Bold values indicate $\mathrm{p}<0.05 .{ }^{\text {a }}$ Data only available from 14 daily and 13 conventional subjects.

FHN Ancillary Study: Comparative Studies of Whole-Body BIA and BIS 
Baseline: 3 times/week $(n=18)$

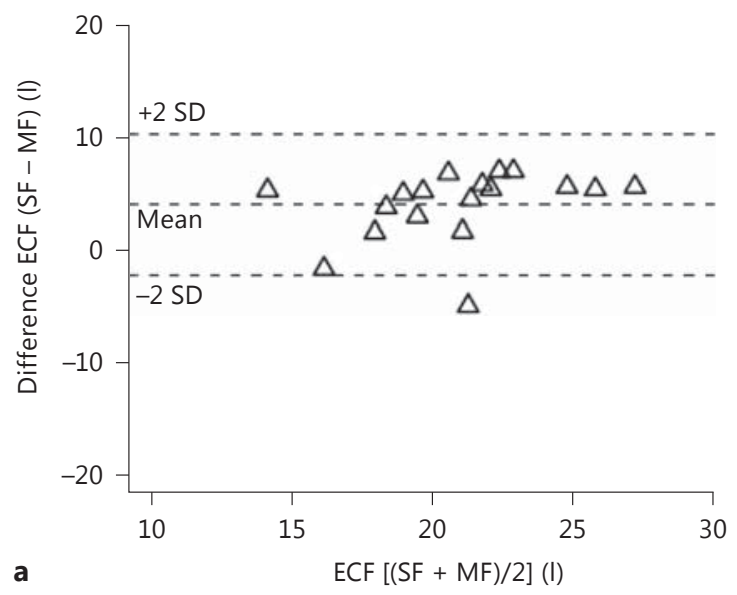

Baseline: 6 times/week $(\mathrm{n}=17)$

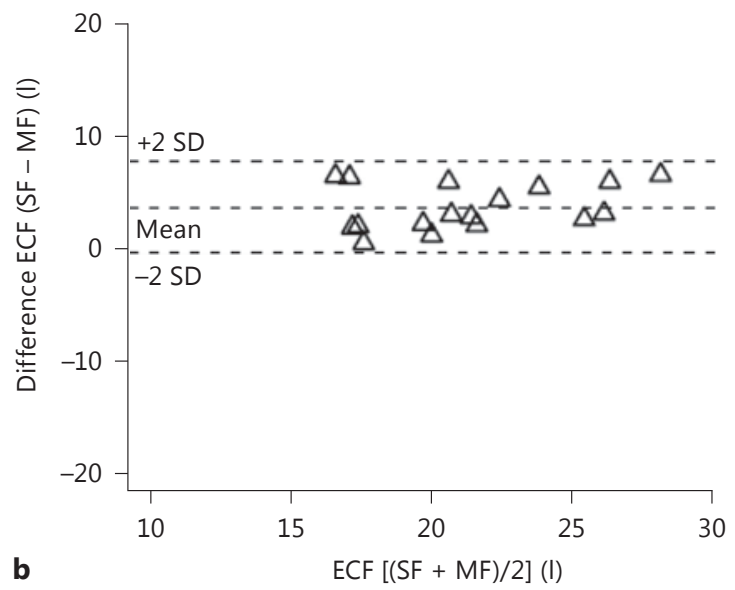

Month 5: 3 times/week $(n=18)$

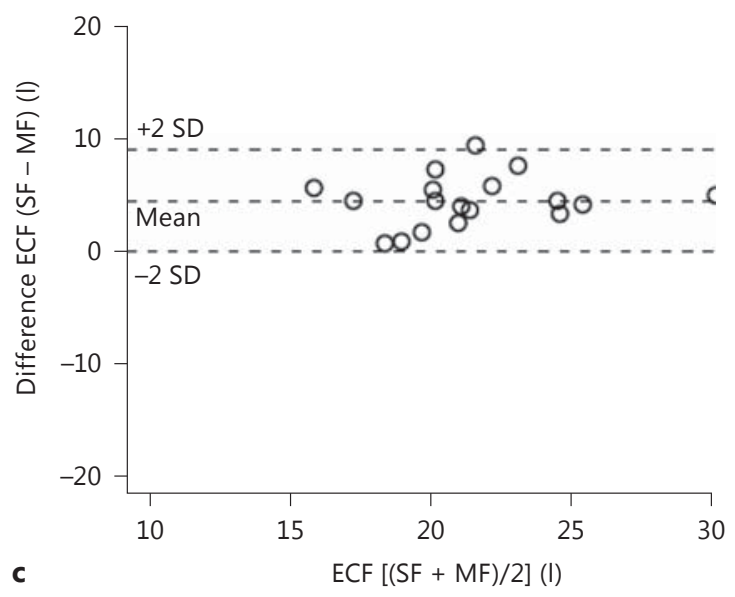

Month 5: 6 times/week $(n=17)$

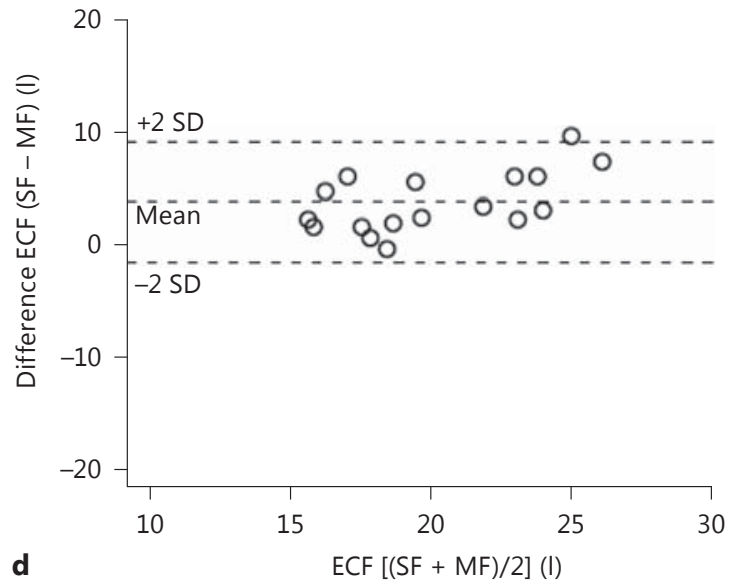

Fig. 6. Bland-Altman plot of ECF as estimated per single- and multi-frequency (SF; MF) bioimpedance in patients randomized in the conventional ( 3 times/week HD; $\mathrm{n}=18$ ) and the interventional (more frequent HD 6 times/ week $\mathrm{HD} ; \mathrm{n}=17)$ arm at baseline $(\mathbf{a}, \mathbf{b})$ and 5 months $(\mathbf{c}, \mathbf{d})$ after randomization.

A significant treatment effect was detected when change was quantified using MF-BIS, which, while similar in magnitude, was not significant when measured with SF-BIA (table 2). Paired analysis of changes in ECF measured by MF-BIA showed a significant treatment effect while SF-BIA did not achieve significance (table 2). PreHD weight did not show any significant changes and no treatment effect. Ultrafiltration volume was significantly lower in those randomized to the more frequent arm due to the shorter interdialytic period.

\section{Power Calculation}

The treatment effect on ECF estimated with MF-BIS was significant $[-1.5(95 \% \mathrm{CI}-2.5$ to -0.5 with an estimated power of $83 \%$ ) l] but not significant with SF-BIA [ $-1.5(95 \% \mathrm{CI}-3.5$ to 0.1$) 1]$ with a probability of $58 \%$ to accept the null hypothesis despite a significant difference being present (type II error) (table 2). Based on these data of ECF, it would have required 86 subjects to be measured with SF-BIA to achieve a statistical power of $80 \%$ (as commonly targeted for clinical trials). 
Table 3. Systematic biases between assessments of TBW, ICF and ECF by SF-BIA and MF-BIS at baseline and at month 5 of the study, and the treatment effect of more frequent $\mathrm{HD}$ (6 times/week)

\begin{tabular}{|c|c|c|c|c|c|}
\hline \multirow[t]{2}{*}{ Parameter } & \multicolumn{2}{|l|}{ Baseline } & \multicolumn{2}{|l|}{ Month 5} & \multirow{2}{*}{$\begin{array}{l}\text { Treatment effect of } \\
6 \text { times/week } \\
\text { HD }\end{array}$} \\
\hline & $\begin{array}{l}\text { conventional HD } \\
\text { (SF-BIA - MF-BIS) }\end{array}$ & $\begin{array}{l}\text { more frequent HD } \\
\text { (SF-BIA - MF-BIS) }\end{array}$ & $\begin{array}{l}\text { conventional HD } \\
\text { (SF-BIA - MF-BIS) }\end{array}$ & $\begin{array}{l}\text { more frequent HD } \\
\text { (SF-BIA - MF-BIS) }\end{array}$ & \\
\hline TBW, 1 & $5.1(3.5$ to 6.7$)$ & $3.9(2.4$ to 5.4$)$ & 4.8 (3.3 to 6.3$)$ & $4(2.5$ to 5.6$)$ & $0.4(-1.8$ to 2.7$)$ \\
\hline ICF, 1 & $1(-1.1$ to 3$)$ & $0.3(-1.2$ to 1.8$)$ & $0.2(-0.8$ to 1.2$)$ & $0.2(-0.7$ to 1.2$)$ & $0.7(-1.5$ to 2.8$)$ \\
\hline $\mathrm{ECF}, 1$ & $4.1(2.7$ to 6.2$)$ & $3.6(2.6$ to 4.5$)$ & $4.6(3.5$ to 5.6$)$ & $3.8(2.5$ to 5.1$)$ & $-0.2(-1.8$ to 1.3$)$ \\
\hline
\end{tabular}

Data on systematic bias and treatment effects reported as mean differences and 95\% CI.

\section{Discussion}

\section{Statement of Principal Findings}

The primary analysis showed that changes in frequency of dialysis treatment, which have been shown to be associated with significant changes in interdialytic weight gain [12] and a decrease in both TBW and ECF as measured by SF-BIA [13], did not affect the agreement (evaluated by Bland-Altman analysis) between TBW, ICF and ECF values estimated by SF-BIA and MF-BIS. Correlations between the assessments using both methods were significant at all time points. Regardless of the limitations of correlational analysis (mainly that the actual coefficient is dependent on the broadness of the range of values) in the context of an analysis testing the agreement between two methods $[18,23]$, the good correlation in concert with a constant systematic error which is independent of the actual value, this analysis suggested that the precision between SF-BIA and MF-BIS is not affected by $\mathrm{HD}$ frequency, and subsequent change in the relative hydration of either the extra- or intracellular compartment. No conclusion on the actual accuracy can be made based on these data, but the treatment comparison clearly shows that treatment allocation did not affect the systematic bias or the precision (reflected by the standard deviations of the measurements) between SF-BIA and MF-BIS.

As a secondary aim, the changes from baseline to month 5 were estimated, the treatment effects were calculated from these changes and compared between the methods. Only MF-BIS was able to capture a borderline significant treatment effect on ECF, while SF-BIA showed only a non-significant trend. Treatment effects on the other fluid volumes were not significant with either method. The lack of statistical power and sufficient precision is likely to be the reason for not finding the results re- ported by Kaysen et al. [13] with all methods. We can conclude from our data that the significant treatment effect on ECF by the intervention seen with the use of MFBIS, but not with the SF-BIA, was a consequence of the higher precision found for MF-BIS as compared to SFBIA in the estimation of ECF. This suggests that the higher level of precision has enabled MF-BIS to detect the treatment effect. Thus for the design of future studies the use of MF-BIS may be beneficial and require a lower recruitment target.

\section{Comparison to Other Studies}

In a randomized cross-over study inducing deviations from euvolemia by either administering Ringer's lactate infusion or a potent loop diuretic, a more accurate assessment (in relation to bromide space assessments) after $2 \mathrm{~h}$ of treatment of volume changes using MF-BIS compared to SF-BIA in 27 healthy subjects was reported [4]. The accuracy of MF-BIS and SF-BIA in detecting intradialytic volume changes compared to ultrafiltration volume as the reference method was recently studied in a non-randomized cohort of Chinese dialysis patients [8]. The precision reported in the results of this study was comparable to our data and the comparison to weight change suggested MF-BIS to be more accurate in detecting shortterm changes in body volumes [8]. A comparable precision of the measurements at all time points and in both treatment arms suggests that, despite the reportedly slightly better accuracy of MF-BIS, SF-BIA appears to be an equally valid and useful tool for longitudinal analyses. Of note, both methods provide non-biased values in measurements of ICF. The difference in the estimation of ECF accounts for the difference observed in the values reported for TBW, whereas ICF shows comparable results. It may also be added that a more recently developed model designed for the use with MF-BIS also incorporating the 
body mass index as an additional input parameter may show improved accuracy and precision and be subject of future studies [24, 25].

One of the major strengths of this analysis is the prospective study design and the randomization of patients after the baseline assessments. There had not been any significant differences between the subject groups suggesting a successful randomization of the studied subjects in the main trial (tables 1,2) [21]. Subjects enrolled in the FHN Daily Trial were reportedly generalizable to the HD population in the United States as per data of the United States Renal Data System [21], which suggests that our data may also be considered generalizable. Furthermore, measurements were done at the same time, location and condition in each of the study sites and also that all repeat measures carried out in the same patients were done with the same device, which excludes the possibility of differences between used devices, electrodes and similar potentially confounding factors.

The main weakness is an inability to draw conclusions on the accuracy of the measurements due to the lack of comparison against dilution methods such as total body potassium and deuterium oxide or an extracellular space marker such as bromide, inulin or iothalamate. The small sample size is an additional weakness.

Our data suggest that body fluid volume and distribution are both affected by increases in HD frequency without altering the agreement between SF-BIA and MF-BIS. In the absence of data from dilution methods it is difficult to judge the accuracy of either method. However, in terms of precision, MF-BIS appears to perform better. This appears to be particularly true for ECF estimation where this technique detected a significant treatment effect which was not detected in the SF-BIA data (despite similar overall changes in volume). The trends found in this analysis are consistent with the data reported by Kaysen et al. [13] showing significant changes in TBW and ECF. The differences in precision of both methods do however affect the power of the analysis. Kaysen et al. [13] reported a treatment effect (month 5 - baseline) on ECF of -1.3 [95\% CI -1.9 to -0.7 with an estimated power of $98 \%(n=134)]$. The comparably lower power which was evident in our data (power of $83 \%$ with MF-BIS and $42 \%$ only with SFBIA) strongly suggests that the lack of significance for SFBIA can entirely be explained by the small sample size. The effects on the power of our analysis were substantial and for SF-BIA the number of patients required to find a treatment effect at a power of $80 \%$ would have been substantially larger for SF-BIA $(n=86)$. These findings may be of particular importance when trials are designed and re- cruitment aims are set. Methods with higher precision and sensitivity for treatment effects of studied interventions may be considered to need fewer enrolled subjects to successfully show effects of the studied intervention. However, the level of precision unaffected by factors resulting from change in frequency such as fluid overload and interdialytic weight gain, confirms the usefulness of either technique in drawing conclusions about changes in TBW and its distribution in prospective longitudinal settings.

The main question of the accuracy of the techniques could not be adequately addressed in this analysis and remains a field for future research. However, this study aimed to assess the usefulness of both techniques for the use in longitudinal settings, which is supported by a good degree of precision unaffected by change in treatment frequency.

\section{Appendix}

\section{Dialysis Clinics of Enrollment}

Renal Research Institute Consortium: Irving Place Dialysis Center, Queens Artificial Kidney Center, South Queens Dialysis Center, Southern Manhattan Hemodialysis Center, St. Alban's Dialysis, Upper Manhattan Dialysis Center, Yorkville Dialysis Center. California Consortium: University of California Davis, DCI University Dialysis Clinic, Sacramento, Calif., USA.

Multi-Frequency Bioelectrical Impedance Spectroscopy

According to the model developed by De Lorenzo et al., based on the Cole-Cole model and the Hanai theory, calculation of intracellular fluid volume $\left(\mathrm{V}_{\mathrm{ICF}}\right)$ [l] and total body fluid (TBW) [1] requires the calculation of extracellular volume $\left(\mathrm{V}_{\mathrm{ECF}}\right)[1]$ as

$$
V_{E C F}=\left(\frac{40.5 K_{B} h e i g h t^{2} \sqrt{\text { weight }}}{1,025 R_{e}}\right)^{\frac{2}{3}}
$$

for men, and

$$
V_{E C F}=\left(\frac{39.0 K_{B} h e i g h t^{2} \sqrt{\text { weight }}}{1,025 R_{e}}\right)^{\frac{2}{3}}
$$

for women,

where $R_{E}$ is the extracellular resistance $[\Omega]$ deriving from model fitting using the Cole-Cole model, height is the height of the subject $[\mathrm{cm}]$, weight the body mass $[\mathrm{kg}]$ and $\mathrm{KB}$ the geometry constant (assumed to be 4.3) $[3,26]$.

Intracellular volume is then calculated as

$$
V_{I C F}=V_{E C F}\left(\left[\frac{\rho_{M I X}\left(R_{i}+R_{e}\right)}{40.5 R_{i}}\right]^{\frac{2}{3}}-1\right)
$$

for men, and 


$$
V_{I C F}=V_{E C F}\left(\left[\frac{\rho_{M I X}\left(R_{i}+R_{e}\right)}{39.0 R_{i}}\right]^{\frac{2}{3}}-1\right)
$$

for women,

where

$$
\rho_{M I X}=273.9-(273.9-40.5)\left(\frac{R_{i}}{R_{i}+R_{e}}\right)^{\frac{2}{3}}
$$

for men, and

$$
\rho_{\text {MIX }}=264.9-(264.9-39.0)\left(\frac{R_{i}}{R_{i}+R_{e}}\right)^{\frac{2}{3}}
$$

for women,

where $R_{e}$ and $R_{i}$ are intra- and extracellular resistance deriving from model fitting using the Cole-Cole model, respectively [3, 26].

In a final step, TBW is then calculated as

$$
\mathrm{TBW}=\mathrm{V}_{\mathrm{ECF}}+\mathrm{V}_{\mathrm{ICF}}
$$

Single-Frequency Bioelectrical Impedance Analysis

According to the model by Kotler et al. [16], TBW can be calculated as

$$
T W B=0.58\left[\frac{L^{1.62}}{Z^{0.70}} \frac{1.0}{1.35}\right]+0.32 \text { weight }-3.66
$$

for men, and

$$
T W B=0.76\left[\frac{L^{1.99}}{Z^{0.58}} \frac{1.0}{18.91}\right]+0.14 \text { weight }-0.86
$$

for women,

where $\mathrm{L}$ is the height $[\mathrm{cm}], \mathrm{Z}$ the electrical impedance, and weight the body mass [kg] [16].

Total body potassium (TBK) [mmol] can be calculated as

$$
T B K=0.76\left[\frac{L^{1.60}}{X_{c p}^{0.50}}(59.06)\right]+18.52 \text { weight }-386.66
$$

for men, and

$$
T B K=0.96\left[\frac{L^{2.07}}{X_{c p}^{0.36}}(1.30)\right]+5.79 \text { weight }-230.51
$$

for women,

where $L$ is the height $[\mathrm{cm}], \mathrm{X}_{\mathrm{cp}}$ the reactance $[\Omega]$, and weight the body mass [kg] [16].

The ICV estimation based on the TBK [ $\mathrm{mmol}]$ is conducted under the assumption that intracellular $\mathrm{K}^{+}$concentration is considerably stable at around $152 \mathrm{mEq} / \mathrm{l}$ and allows, in the knowledge of TBW, the estimation of the intracellular $\mathrm{K}^{+}$distribution volume [17].

\section{Acknowledgements}

The authors thank the entire Frequent Hemodialysis Network (FHN) Study Group. Parts of the study were presented at the Renal Week 2009 of the American Society of Nephrology in San Diego, Calif., USA, and at the XLVIII ERA-EDTA Congress in Prague, Czech Republic. The project described was supported in part by the National Center for Research Resources, National Institutes of Health (through grant No. UL1 RR024146), by the RRI and by the University of California Davis Clinical and Translational Science Center NIH grant No. RR 0024146.

\section{Disclosure Statement}

Peter Kotanko and Nathan W. Levin hold stock in Fresenius Medical Care NA. All other authors do not have any potential financial conflicts of interest to declare. The authors hereby declare that the results presented in this paper have not been published previously in whole or part, except in abstract format at the Renal Week 2009 of the American Society of Nephrology in San Diego, Calif., USA, and at the XLVIII ERA-EDTA Congress in Prague, Czech Republic.

\section{References}


-9 Jaffrin MY, Morel H: Body fluid volumes measurements by impedance: a review of bioimpedance spectroscopy (BIS) and bioimpedance analysis (BIA) methods. Med Eng Phys 2008;30:1257-1269.

10 Carter M, Morris AT, Zhu F, Zaluska W, Levin NW: Effect of body mass index on estimation of extracellular volume) in hemodialysis patients using segmental and wholebody bioimpedance analysis. Physiol Meas 2005;26:S93-S99.

-11 Abbas SR, Zhu F, Kaysen GA, Kotanko P, Levin NW: Effect of change in fluid distribution in segments in hemodialysis patients at different ultrafiltration rates on accuracy of whole-body bioimpedance measurement. J Appl Physiol 2014;116:1382-1389.

12 Chertow GM, Levin NW, Beck GJ, Depner TA, Eggers PW, Gassman JJ, Gorodetskaya I, Greene T, James S, Larive B, Lindsay RM, Mehta RL, Miller B, Ornt DB, Rajagopalan S, Rastogi A, Rocco MV, Schiller B, Sergeyeva O, Schulman G, Ting GO, Unruh ML, Star RA, Kliger AS: In-center hemodialysis six times per week versus three times per week. N Engl J Med 2010;363:2287-2300.

13 Kaysen GA, Greene T, Larive B, Mehta RL, Lindsay RM, Depner TA, Hall YN, Daugirdas JT, Chertow GM: The effect of frequent hemodialysis on nutrition and body composition: Frequent Hemodialysis Network trial. Kidney Int 2012;82:90-99.
14 Sergeyeva O, Gorodetskaya I, Ramos R, Schiller BM, Larive B, Raimann JG, Ting GO, Eggers PW, Chertow GM, Levin NW, Frequent Hemodialysis Network Trials G: Challenges to enrollment and randomization of the Frequent Hemodialysis Network (FHN) Daily Trial. J Nephrol 2012;25:302-309.

15 Suri RS, Garg AX, Chertow GM, Levin NW, Rocco MV, Greene T, Beck GJ, Gassman JJ, Eggers PW, Star RA, Ornt DB, Kliger AS: Frequent Hemodialysis Network (FHN) randomized trials: study design. Kidney Int 2007; 71:349-359.

16 Kotler DP, Burastero S, Wang J, Pierson RN Jr: Prediction of body cell mass, fat-free mass, and total body water with bioelectrical impedance analysis: effects of race, sex, and disease. Am J Clin Nutr 1996;64:489S-497S.

17 Wang Z, St-Onge MP, Lecumberri B, Pi-Sunyer FX, Heshka S, Wang J, Kotler DP, Gallagher D, Wielopolski L, Pierson RN Jr, Heymsfield SB: Body cell mass: model development and validation at the cellular level of body composition. Am J Physiol Endocrinol Metab 2004;286:E123-E128.

18 Bland JM, Altman DG: Statistical methods for assessing agreement between two methods of clinical measurement. Lancet 1986;1:307-310.

19 Bland JM, Altman DG: Measurement error proportional to the mean. BMJ 1996;313:106.

20 Kaysen GA, Larive B, Painter P, Craig A, Lindsay RM, Rocco MV, Daugirdas JT, Schulman G, Chertow GM: Baseline physical performance, health, and functioning of participants in the Frequent Hemodialysis Network (FHN) trial. Am J Kidney Dis 2010;57:101-112.
21 Rocco MV, Larive B, Eggers PW, Beck GJ, Chertow GM, Levin NW, Kliger AS: Baseline characteristics of participants in the Frequent Hemodialysis Network (FHN) daily and nocturnal trials. Am J Kidney Dis 2010;57:90-100.

22 Daugirdas JT, Greene T, Rocco MV, Kaysen GA, Depner TA, Levin NW, Chertow GM, Ornt DB, Raimann JG, Larive B, Kliger AS: Effect of frequent hemodialysis on residual kidney function. Kidney Int 2013;83:949-958.

23 Van Stralen KJ, Jager KJ, Zoccali C, Dekker FW: Agreement between methods. Kidney Int 2008;74:1116-1120.

24 Moissl UM, Wabel P, Chamney PW, Bosaeus I, Levin NW, Bosy-Westphal A, Korth O, Muller MJ, Ellegard L, Malmros V, Kaitwatcharachai C, Kuhlmann MK, Zhu F, Fuller NJ: Body fluid volume determination via body composition spectroscopy in health and disease. Physiol Meas 2006;27:921-933.

25 Chamney PW, Wabel P, Moissl UM, Muller MJ, Bosy-Westphal A, Korth O, Fuller NJ: A whole-body model to distinguish excess fluid from the hydration of major body tissues. Am J Clin Nutr 2007;85:80-89.

26 Matthie JR: Second-generation mixture theory equation for estimating intracellular water using bioimpedance spectroscopy. J Appl Physiol 2005;99:780-781. 